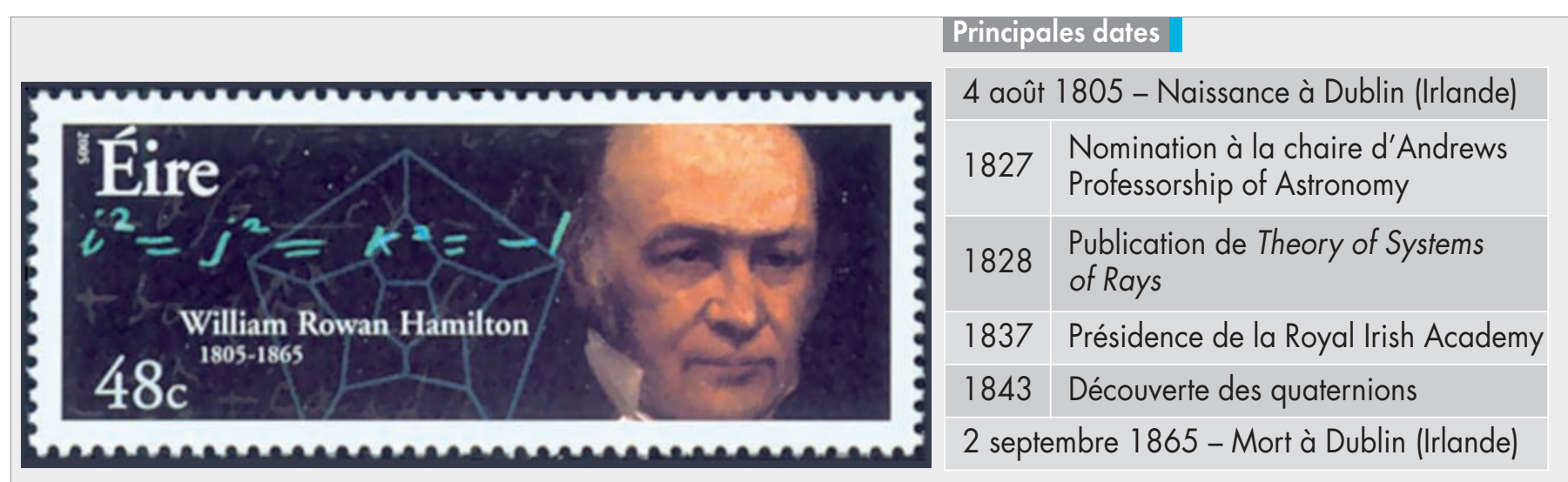

\title{
Sir William Rowan Hamilton
}

\section{Riad Haidar}

haidar@onera.fr

Mathématicien, physicien et astronome, William Rowan Hamilton est une figure emblématique du génie irlandais. Il est particulièrement connu pour sa découverte des quaternions et ses recherches sur les opérateurs linéaires. Par ailleurs, ses travaux en dynamique se révéleront décisifs pour le développement de la théorie classique des champs, ainsi que pour l'essor de la mécanique quantique près d'un siècle après sa mort.

W illiam Rowan Hamilton naît le 4 août 1805 dans une famille aisée de Dublin, sur la côte est de l'Irlande. Son père Archibald est un solicitor (avocat non plaidant), très occupé par sa fonction et souvent absent du foyer familial. Sa mère Sarah Hutton est un esprit brillant. Elle décèle très tôt chez William une grande vivacité intellectuelle et décide de le confier, dès l'âge de trois ans, à son oncle, le Révérend James Hamilton. Linguiste notoire formé au prestigieux Trinity College, James vit dans le magnifique manoir du Talbot Castle dans le village de Trim, au nord de Dublin. II développe les dons naturels du jeune William, qui maîtrise vite une douzaine de langues, mortes et vivantes.

Hamilton se découvre également un certain talent et du goût pour I'arithmétique. À dix ans, on le présente au jeune prodige américain du calcul mental Zerah Colburn, alors de passage à Dublin - et il réussit le tour de force de donner le change ! Son intérêt pour les mathématiques s'affirme, et il dévore littéralement les ouvrages classiques et modernes: I'Algèbre de Clairaut, les Éléments d'Euclide et l'Arithmetica universalis de Newton.

En 1821, âgé de 16 ans, Hamilton est admis au Trinity College de Dublin. Dès la première année, il décroche un optime pour ses résultats en grec et latin. C'est une distinction rare et prestigieuse, qui couronne l'enseignement de l'oncle James. L'été suivant, il entreprend l'étude systématique de la Mécanique Céleste : I'ouvrage de Laplace foisonne de nouveaux et subtils procédés analytiques, que le jeune Hamilton décortique soigneusement, parfois laborieusement. Mais il perçoit toute la richesse de l'héritage du grand maître, et la greffe prend. Hamilton décide de se consacrer désormais à la recherche en mathématiques.

\section{Premiers travaux}

En août 1824, Hamilton rencontre Catherine Disney : c'est un coup de foudre. Mais il est encore étudiant et, surtout, elle est promise à Barlow, un clergyman à la situation déjà bien établie. Le jeune homme ne peut rivaliser. L'esprit pétri des légendes chevaleresques qui ont bercé son enfance au Talbot Castle, Hamilton accuse durement le coup : ses notes et sa santé déclinent, il se réfugie dans la poésie - une habitude qui lui restera dans les moments de peine.

Mais il parvient à se ressaisir. Quelques mois plus tard, il soumet son premier papier à la Royal Irish Academy. L'accueil est courtois : les rapporteurs relèvent des insuffisances et réclament des éclaircissements. Hamilton s'y plie, affine ses arguments. En 1828, I'article parait enfin sous le titre Theory of Systems of Rays. II est le premier volet d'une série $d^{\prime}$ 'articles devenus célèbres sur le 
principe variationnel, aussi appelé Principe de Hamilton. Ce principe, reformulé par Jacobi, aboutit à une formulation alternative de la mécanique classique, désormais connue sous le nom de mécanique hamiltonienne. Cette formulation, comme la mécanique lagrangienne sur laquelle elle est fondée, fournit une méthode extraordinairement puissante pour résoudre les équations du mouvement et est aujourd'hui utilisée en électromagnétisme et en mécanique quantique.

Entre-temps, il poursuit sa trajectoire fulgurante au Trinity College. Systématiquement classé premier à toutes les épreuves dans
Here as he walked by on the loth of October 1843 Sir William Rowan Hamilton in a flash of genius discovered the fundamental formula for quaternion multiplication $i^{2}=j^{2}=k^{2}=i j k=-1$

$\varepsilon$ cut it on a stone of this bridge de la Royal Irish Academy. La solution lui apparaît soudain, sous la forme $i^{2}=i^{2}=k^{2}=i j k=-1$. Enthousiaste, il grave ces équations au couteau dans une pile du pont de Broom Bridge. C'est l'acte de naissance des quaternions, le premier algèbre non commutatif de l'Histoire. La formule gravée en 1843 a disparu, mais une plaque commémore ce geste.

Malgré ce succès, la période qui suit est particulièrement éprouvante. En 1845, il reçoit la visite amicale de Thomas Disney et de sa fille Catherine. Ces retrouvailles l'anéantissent. II sombre si profondément dans l'alcool que plusieurs amis s'en émeutoutes les matières, il décroche en 1827, avant même la fin de ses études, la chaire d'Andrews Professorship of Astronomy de l'université de Dublin. II s'installe alors, Royal Astronomer of Ireland de tout juste 22 ans, au Dunsink Observatory. II y poursuit ses recherches en mathématiques appliquées. En 1832, il publie son troisième supplément à la Theory of Systems of Rays où il applique sa fonction caractéristique à l'optique et aux surfaces d'onde de Fresnel, et prévoit ainsi la réfraction conique.

Sa chère Catherine ayant épousé Barlow et lui étant désormais inaccessible, Hamilton se montre particulièrement gauche et malheureux dans ses relations avec les femmes. En 1832 il finit par épouser Helen Maria Bayly, une jeune fille frustre d'une ferme voisine de l'Observatoire. Ils auront deux garçons et une fille. Pourtant cette union sans passion, entre deux êtres qui se révèlent peu compatibles, laisse à Hamilton un sentiment persistant d'amertume. Helen, qui est d'une nature maladive, s'absente souvent et longuement, parfois pendant plusieurs mois, de l'Observatoire, y abandonnant époux et enfants. Hamilton, incapable de s'adapter à cette solitude récurrente, prend l'habitude de chercher un réconfort dans la boisson. La situation devient si désespérée que sa sœur Eliza décide de le rejoindre, et s'installe à Dunsink.

\section{Les quaternions}

En 1833, Hamilton a l'idée d'exprimer les nombres complexes sous la forme de couples algébriques (paires ordonnées de nombres réels). Il tente ensuite d'étendre ce formalisme séduisant à des dimensions supérieures, et d'abord aux triplets. Cette idée l'obsède de longues années, au point que même ses enfants s'en rendent compte et l'accueillent tous les matins par la question rituelle : "Alors, papa, sais-tu comment multiplier les triplets? », à laquelle il répond invariablement qu'il ne parvient qu'à les additionner et les soustraire. L'effort intellectuel durera près de dix ans. En vain, et pour cause : Frobenius démontrera en 1877 que c'est impossible.

Hamilton a plus de succès avec les quadruplets. L'anecdote est entrée dans la légende : le 16 octobre 1843, il marche le long du Canal Royal de Dublin avec sa femme, en route vers une soirée vent, et lui conseillent une abstinence totale. Mais les événements tragiques s'enchaînent, et ont raison de sa volonté. En 1847 son cher oncle James décède. En 1848 Catherine entame une correspondance nourrie, qui prend une tournure si intime que, atteinte de remords, elle s'en confesse à son époux et tente de se suicider. Elle finit par se réfugier chez sa mère, sans pour autant divorcer... Tout ceci fragilise Hamilton, qui se retrouve dans une situation personnelle d'extrême précarité. Pourtant, comme toujours, il parvient à maintenir une forte activité intellectuelle. II entreprend la rédaction de l'imposant Lectures on Quaternions, qu'il achève en 1853 mais qui s'avère un échec pédagogique. Décidé à laisser une œuvre qui lui survive, Hamilton entame la rédaction d'un second livre sur les quaternions, sur le modèle des Eléments d'Euclide. Ce livre, qu'il laisse inachevé, est publié à titre posthume avec une préface de son fils William Edwin Hamilton.

\section{Ultima verbae}

Hamilton est un scientifique tenace, qui nourrit un goût pour la perfection. Son œuvre est immense, et déborde largement les livres et articles qui nous sont parvenus. II a notamment étudié les solutions algébriques des équations du cinquième degré et on lui doit l'invention ingénieuse de l'hodographe.

II reçoit de nombreux honneurs. En 1835, il est anobli par le Lord Lieutenant de Dublin. Cette même année, il est lauréat de la Royal Medal. En 1837, il est élu Président de la Royal Irish Academy, et membre correspondant de l'académie de Saint-Petersbourg. Il est aussi le premier membre étranger de la National Academy of Sciences des États-Unis.

Hamilton s'éteint le 2 septembre 1865, des suites d'une sévère attaque de goutte. Il est enterré au cimetière de Mount Jerome à Dublin.

\section{Référence}

Sir William Rowan Hamilton, Encyclopédie MacTutor, University of St Andrews. 